\title{
Reabilitação estética anterior associando prótese metalocerâmica e prótese fixa metal-free: relato de caso
}

\author{
Previous aesthetic rehabilitation associated with metaloceramic prosthesis and fixed metal-free prosthesis: case report \\ Rehabilitación estética anterior asociando prótesis metalocerámica y prótesis fixa metal-free: reporte de caso \\ Juliana Lujan BRUNETTO \\ Marcio CAMPANER \\ Caroline de Freitas JORGE \\ Letícia Cerri MAZZA \\ Sandro Basso BITENCOURT \\ Adriane Boaventura CHIORLIN \\ Ricardo SHIBAYAMA \\ Aldiéris Alves PESQUEIRA
}

Departamento de Materiais Odontológicos e Prótese, Faculdade de Odontologia de Araçatuba, FOA-UNESP Univ. Estadual Paulista, 16015-050 Araçatuba SP, Brasil Departamento de Odontologia Restauradora, Centro de Ciências da Saúde, Universidade Estadual de Londrina - CCS-UEL, 86057-970, Londrina - PR, Brasil

\section{Resumo}

Devido a evolução dos sistemas cerâmicos, atualmente, são permitidas inúmeras associações de materiais restauradores. Entretanto, mimetizar as características visuais das próteses livres de metal com próteses metalocerâmica permanece um desafio. Assim, o objetivo deste trabalho foi relatar o caso clínico de substituição de próteses desadaptadas, homogeneizando próteses parciais fixas livres de metal e metalocerâmicas dento e implantossuportadas, após cirurgia periodontal. O paciente de 55 anos, gênero masculino, compareceu à Clínica de Prótese Parcial Fixa, da Faculdade de Odontologia de Araçatuba - FOA/UNESP em 2017 queixando-se dos seus dentes anteriores estarem "feios" e com a "raiz aparecendo". No exame clínico foi constatada recessão gengival nos elementos 11 e 13, os quais continham uma prótese parcial fixa de três elementos metalocerâmicas desadaptada, prótese sobre implante nos elementos 21 e 22, que apresentavam-se desadaptadas e com estética desfavorável. No exame radiográfico pôde-se observar a presença de um núcleo metálico fundido nos elementos 11 e 13. Foi proposto, então, a confecção de coroas totais livres de metal nos elementos anteriores superiores $(13,12,11,21$ e 23) e coroa metalocerâmica para o elemento 22 . Para isso, foi realizado enceramento diagnóstico inicial e, após o consentimento do paciente, foi efetuada a remoção das próteses antigas. Com a remoção, visualizamos uma depressão na vestibular do rebordo na área do 12 (ausente), solucionado com um enxerto gengival subepitelial. Após a realização dos preparos dentários e moldagem com silicone de adição as peças foram confeccionadas com sistema e.max Ceram (Ivoclar Vivadent). Após a prova estética e ajustes oclusais iniciais, as peças foram preparadas para cimentação resinosa com o sistema Variolink® N (Ivoclar Vivadent). O cimento transparente foi selecionado previamente por meio de provas com o kit Try-In. Conclui-se que, apesar da disparidade dos materiais, é possível mimetizá-los e oferecer resultados reabilitadores suficientemente estéticos e satisfatórios.

Descritores: Implantação Dentária; Estética Dentária; Materiais Dentários.

\section{Abstract}

Due to the evolution of ceramic systems, numerous associations of restorative materials are now permitted. However, mimicking the visual characteristics of metal-free prostheses with metal-ceramic dentures remains a challenge. Thus, the objective of this study was to report the clinical case of replacement of maladaptive dentures, homogenizing denture free and fixed metal partial dentures and implants supported after periodontal surgery. The patient, 55 years old, male, attended the Fixed Partial Prosthesis Clinic of the Faculty of Dentistry of Araçatuba - FOA / UNESP in 2017 complaining that his anterior teeth were "ugly" and with the "root appearing". In the clinical examination, gingival recession was observed in elements 11 and 13, which contained a fixed partial prosthesis of three maladaptive metal-ceramic elements, a prosthesis on implants in elements 21 and 22, the prostheses were maladapted and unfavorable esthetics. In the radiographic examination it is possible to observe the presence of a molten metal nucleus in elements 11 and 13. It was proposed, then, the manufacture of free metal crowns in the upper anterior elements $(13,12,11,21$ and 23) and crown PFM for the element 22. For this, it performed waxing initial diagnosis and after the patient's consent, the removal of the old prosthesis were performed. Upon removal, a depression visualize the buccal flange in the area 12 (absent), solved with a subepithelial gum graft. After the dental preparations and molding with silicone of addition the pieces were made with e.max Ceram system (Ivoclar Vivadent). After the initial test and aesthetic occlusal adjustments, the pieces were prepared for cementing with resin Variolink® N system (Ivoclar Vivadent). The clear cement was previously selected by the test realized with Try-In kit. We conclude that, despite the disparity of materials, it is possible to mimic them and offer rehabilitative results sufficiently aesthetic and satisfactory.

Descriptors: Dental Implantation; Dental Esthetics; Dental Materials.

\section{Resumen}

Debido a la evolución de los sistemas cerámicos, actualmente, se permiten numerosas asociaciones de materiales restauradores. Sin embargo, mimetizar las características visuales de las prótesis libres de metal con prótesis metalocerámicas sigue siendo un desafío. Así, el objetivo de este trabajo fue relatar el caso clínico de sustitución de prótesis desadaptadas, homogeneizando prótesis parciales fijas libres de metal y metalocerámicas dento e implantosuportadas, después de cirugía periodontal. El paciente de 55 años, género masculino, asistió a la Clínica de Prótesis Parcial Fija, de la Facultad de Odontología de Araçatuba - FOA / UNESP en 2017 quejándose de sus dientes anteriores estar "feos" y con la "raíz apareciendo". En el examen clínico se constató recesión gingival en los elementos 11 y 13, los cuales contenían una prótesis parcial fija de tres elementos metalocerámicos desadaptados, prótesis sobre implante en los elementos 21 y 22, las prótesis se presentaban desadaptadas y con estética desfavorable. En el examen radiográfico se puede observar la presencia de un núcleo metálico fundido en los elementos 11 y 13 . Se propuso entonces la confección de coronas totales de metal en los elementos anteriores superiores $(13,12,11,21$ y 23) y corona metalocerámica para el elemento 22. Para ello, se realizó el encerado diagnóstico inicial y, tras el consentimiento del paciente, se efectuó la remoción de las prótesis antiguas. Con la remoción, visualizamos una depresión en la vestibular del borde en el área del 12 (ausente), solucionado con un injerto gingival subepitelial. Después de la realización de los preparados dentales y moldeo con silicona de adición las piezas fueron confeccionadas con sistema e.max Ceram (Ivoclar Vivadent). Después de la prueba estética y ajustes oclusales iniciales, las piezas fueron preparadas para cementación resinosa con el sistema Variolink® N (Ivoclar Vivadent). El cemento transparente fue seleccionado previamente por medio de pruebas con el kit Try-In. Se concluye que, a pesar de la disparidad de los materiales, es posible mimetizarlos y ofrecer resultados rehabilitadores suficientemente estéticos y satisfactorios. Descriptores: Implantación Dental; Estética Dental; Materiales Dentales.

\section{INTRODUÇÃO}

A crescente valorização da harmonização facial, incluindo um sorriso esteticamente agradável, faz com que os pacientes busquem cada vez mais alternativas de procedimentos estéticos ${ }^{1,2,3}$. O tratamento adequado das imperfeições dentárias depende da condição clínica de cada paciente, do conhecimento do operador, dos materiais empregados e das técnicas disponíveis ${ }^{2,4-7}$. 
Para que as reabilitações orais restabeleçam adequadamente a estética e a função com implantes e próteses, é essencial que o operador siga o correto posicionamento tridimensional e que o primeiro osseointegre $^{8-10}$. Porém, devido aos diversos componentes intermediários disponíveis no mercado e o posicionamento dos implantes, faz-se necessária a utilização de diversos materiais para a confecção das próteses implantossuportadas. Juntamente com os pilares para Prótese Parcial Fixa (PPF) e seus materiais, a dificuldade de posicionamento e regularização da cor e formato destas afeta esses casos clínicos considerando-os desafiadores. A escolha do material utilizado influencia no resultado estético, na longevidade e na integridade marginal $^{11-13}$, agravando ou não seu prognóstico ${ }^{14}$.

A alta demanda estética, já citada, fez com que as próteses livres de metal se difundissem no mercado, pois ao compararmos os materiais disponíveis, as coroas metalocerâmicas frequentemente apresentam um alo acinzentado devido a presença do coping metálico, afetando sua aparência ${ }^{15}$. Ao realizar um tratamento reabilitador com ambos os materiais, a complexidade desse é elevada pois a translucidez apresentada pelas próteses livres de metal e metalocerâmicas são significantemente diferentes, principalmente, em região anterior ${ }^{16}$. Além dos materiais, a relação das coroas protéticas com o tecido periodontal também afeta o resultado final, uma vez que próteses dentossuportadas e implantossuportadas apresentam comportamentos periodontais diferentes. Para uma boa mimetização das características periodontais em reabiliatações implantossuportadas é importante que o implante seja inserido seguindo a regra tridimensional do seu posicionamento, de acordo com o planejamento e guia cirúrgico. Porém, devido à sua natureza, esses sofrem adaptação morfológica e funcional dos tecidos cervicais peri-implantares, apresentando reabsorções ósseas que podem resultar em black spaces entre dente e implante ${ }^{17,18}$.

O propósito deste trabalho foi relatar caso clínico em que foi necessário realizar a substituição de PPFs desadaptadas, associando metalocerâmica e livres de metal, dento e implantossuportadas, após cirurgia de enxerto conjuntivo.

\section{CASO CLÍNICO}

Paciente, gênero masculino, 55 anos, compareceu à Clínica de Prótese Parcial Fixa (PPF) da Faculdade de Odontologia de Araçatuba, da Universidade Estadual Paulista "Júlio de Mesquita Filho" (FOA-UNESP) em 2017, queixando-se dos seus dentes anteriores estarem "feios" e com a "raiz aparecendo". Após anamnese e exame clínico detalhado, foi verificada a presença de PPF metalocerâmica do 11 ao 13, apresentando desadaptação marginal nos pilares. Ademais, o paciente apresentava coroa unitária metalocerâmica no dente 23 e sobre implante no 21 e 22. Para o correto diagnóstico, o paciente foi submetido à moldagem com silicone de condensação denso Zetaplus e leve (light) Oranwash (Zhermack, Badia Polesine, Itália) em passo único com técnica da dupla mistura, aguardando-se a presa do material de acordo com as recomendações do fabricante. Após a confecção dos modelos em gesso especial tipo IV (Durone - Dentsply Sirona, York, EUA), foi realizada montagem em articulador semi-ajustável (A7-Plus, Bioart, SP, Brasil) e enviado ao laboratório para o enceramento diagnóstico dos dentes anteriores superiores. Após diagnóstico no exame clínico e realização do mock up, foi proposta substituição das próteses de canino a canino superiores. Para isso, foi realizado exame fotográfico extra e intraoral (Figuras 1 e 2) e exame radiográfico para análise da implantação óssea das raízes, verificando a presença de tratamento endodôntico e núcleo nos dentes 11,13 e 23. Inicialmente, as próteses antigas foram removidas, o paciente recebeu anestesia infiltrativa local com anestésico de $2 \%$ de cloridrato de mepivacaína e adrenalina 1:100.000 (Dentisply, York, EUA) e realizada secção das coroas protéticas dos elementos 11 e 13 com ponta diamantada 3216 (JOTA, Rüthi, Suíça) e, ao chegar ao coping metálico, essa foi trocada pela broca transmetal (JOTA, Rüthi, Suíça), até se observar a linha de cimentação, permitindo a completa remoção das próteses. Posteriormente, os pilares foram repreparados com ponta diamantada 4138 (JOTA, Rüthi, Suíça) em alta rotação e irrigação constante. Os provisórios foram previamente confeccionados pela técnica da prensagem laboratorial, em resina acrílica termopolimerizável (Figura 3), reembasados pela técnica de Nealon ${ }^{19}$, avaliando sua anatomia, cor, formato, adaptação e oclusão. Os ajustes necessários foram realizados com auxílio de micromotor (Kavo, Joinville, SC, Brasil) em baixa rotação e com broca de tungstênio Maxicut (PM Edenta, AU/SG, Suíça).

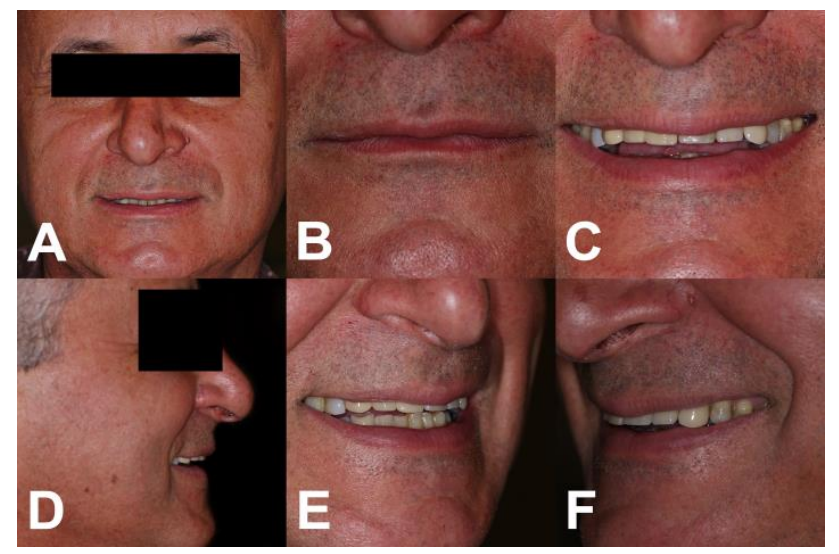

Figura 1: Fotografias extraorais para planejamento. A: Frontal aproximado sorrindo. B: Frontal aproximado com lábios em repouso. C: Frontal aproximado sorrindo. D: Lateral $\left(90^{\circ}\right)$ sorrindo. E: Lateral $\left(45^{\circ}\right)$ direita sorrindo. F: Lateral $\left(45^{\circ}\right)$ esquerda sorrindo. 


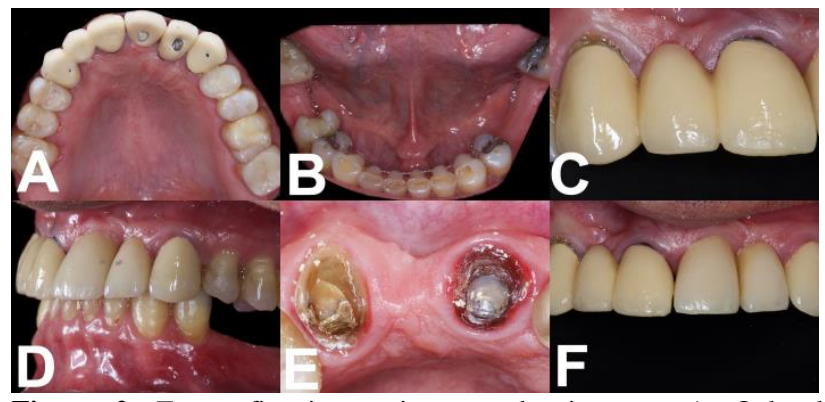

Figura 2: Fotografias intraorais para planejamento. A: Oclusal superior. B: Oclusal inferior. C: Desadaptação das próteses dos dentes 11 e 13. D: Lateral $\left(90^{\circ}\right)$ direita. E: Depressão vestibular na área do 12. F: Frontal superior inicial.

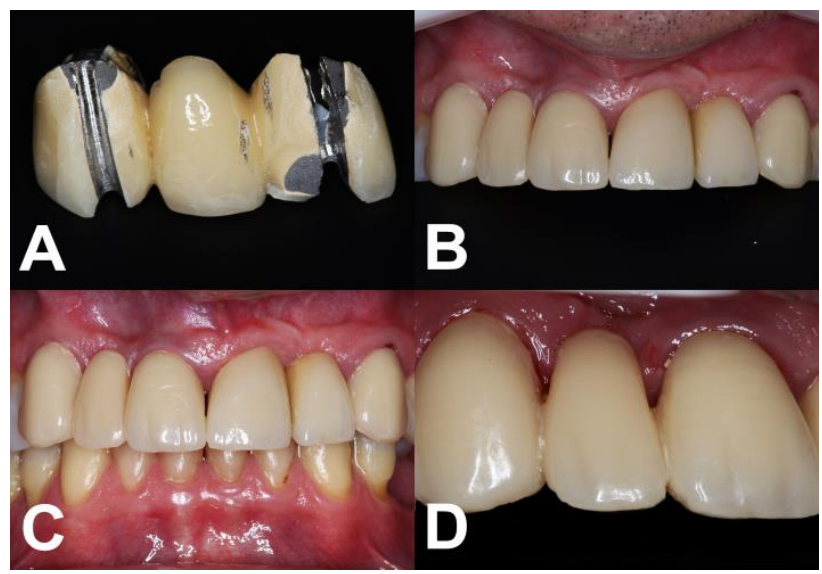

Figura 3: Remoção da prótese e provisionalização imediata. A: Prótese removida. B: Frontal com provisórios cimentados. C: Provisórios em oclusão. D: Adaptação dos provisórios.

$\mathrm{Na}$ sequência, foi realizado o acabamento para remoção das irregularidades das bordas e/ou excessos com o Kit Polimento de PPF UNESP,Araçatuba(Sistemas Dh Pro Curitiba, Brasil), seguindo a sequência preconizada pela disciplina de PPF da UNESP Araçatuba e cimentados com o cimento Rely X Temp NE (3M ESPE, MN, EUA).

Após a provisionalização, o paciente foi submetido à cirurgia de enxerto conjuntivo na vestibular do incisivo lateral superior direito, passando pelo preparo prévios já padronizados na literatura $^{20}$. Previamente, foi anestesiado com anestésico de $2 \%$ de cloridrato de mepivacaína e adrenalina 1:100.000 (Dentisply, York, EUA), incisado o centro do rebordo do 12 com lâmina de bisturi estéril $\mathrm{n}^{\circ} 15 \mathrm{c}$ e cabo $\mathrm{n}^{\circ} 3$ (Figura 4), feito descolamento parcial com o descolador de periósteo de Molt $\mathrm{n}^{\circ} 9$ confeccionando um envelope ${ }^{21}$. Para a remoção do enxerto conjuntivo, foi realizada a técnica do alçapão, sendo o palato a área doadora, onde foi necessário rebater o retalho total para sua remoção. O tecido removido foi, então, posicionado no envelope vestibular e suturado em planos, com pontos simples, utilizando-se o fio Vicryl 6-0 de Poliglactina 910 (ETHICON, Johnson \& Johnson, NJ, EUA). Ao término da cirurgia, foi possível verificar uma melhora significativa da depressão na região vestibular do rebordo. As PPFs provisórias foram ajustadas com broca de tungstênio Maxicut, liberando a área de qualquer pressão exercida (Figura 5), por essas e instaladas para aguardar o período de 90 dias para a completa cicatrização. O paciente foi medicado e foram passadas as recomendações pós-operatórias.

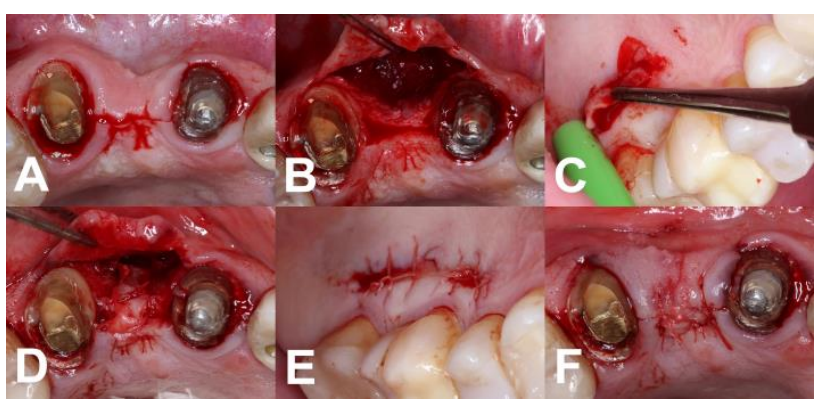

Figura 4: Cirurgia periodontal em tecido mole. A: Incisão no rebordo. B: Retalho descolado em envelope por vestibular. C: Incisão em palato. D: Posicionamento e sutura do enxerto conjuntivo. E: Sutura na área doadora. F: Sutura na área receptora.

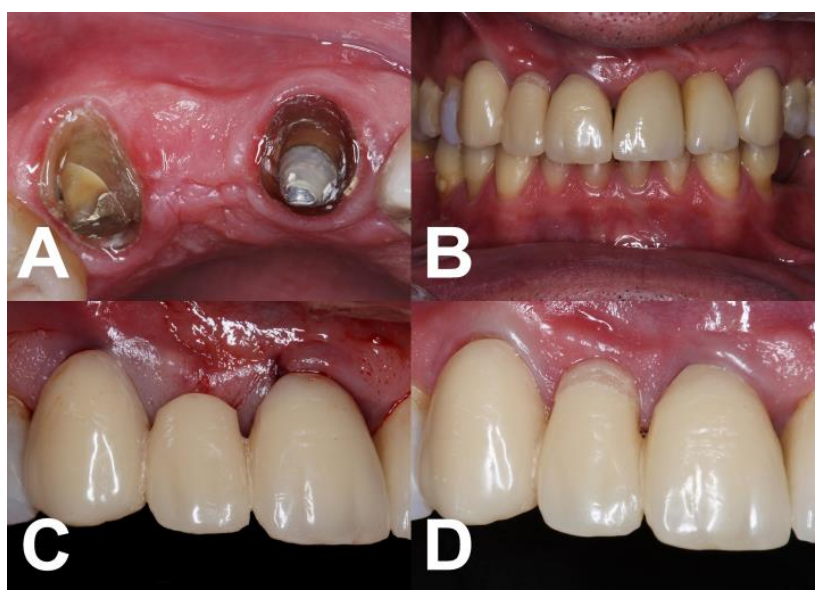

Figura 5: Provisórios e cicatrização do tecido periodontal. A Instalação do provisório após a cirurgia. B: Início do condicionamento gengival. C: Provisório condicionando o tecido gengival em oclusão. D: Tecido gengival após enxerto conjuntivo e cicatrização.

Após a cicatrização, foi removida a prótese provisória do 11-13, para o condicionamento gengival na região do elemento 12. Para isto, foi acrescentada resina acrílica autopolimerizável (Duralay, Reliance Dental Mfg CO, Worth, IL, EUA) na cervical do provisório, pela técnica de Nealon, em três momentos durante um mês. Ao término do condicionamento, as próteses sobre implante do $21 \mathrm{e}$ 22 e a coroa metalocerâmica do dente do 23 (Figura 6) foram removidas e, então, realizados os preparos para coroa total. Devido ao posicionamento do implante no 21, não foi possível confeccionar uma coroa metal free e optou-se por uma coroa metalocerâmica com componente intermediário Esteticone (TitaniumFix, São José dos Campos, SP, Brasil).

Como na Figura 7, para a moldagem, os componentes de transferência foram posicionados e indexados com resina acrílica Duralay (Reliance Dental Mfg CO, Worth, IL, EUA). Já nos pilares protéticos, foi utilizada a técnica do afastamento gengival mecânico-químico com duplo fio retrator 
(Ultrapack, Ultradent, SP, Brasil), sendo embebido o fio\#000 em cloreto de alumínio (Hemostop -Dentsply Sirona, York, EUA) para a obtenção dos copings. Na introdução intrassulcular dos fios, o primeiro (\#000), e de menor diâmetro, teve a função de reduzir o fluxo gengival e o segundo ou mais calibroso, efetivou o afastamento gengival e expôs o término dos preparos dos dentes 11,13 e 23 .

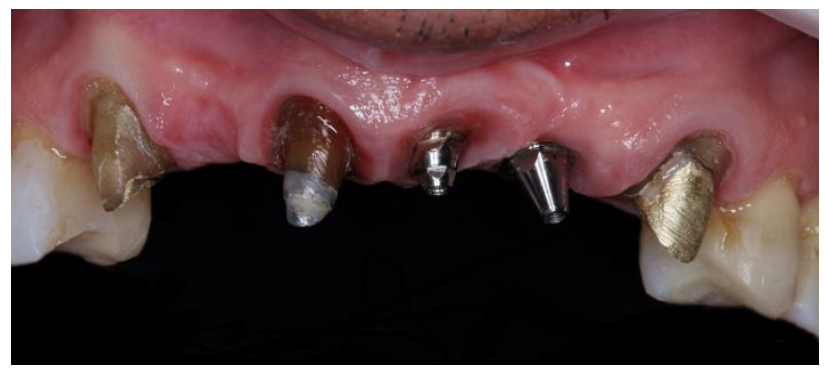

Figura 6: Pilares e componentes protéticos sobre implante em maxila.

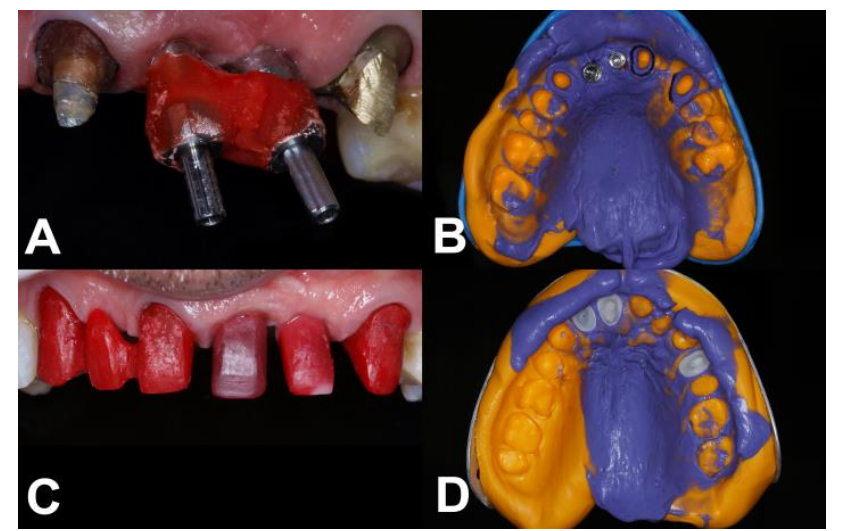

Figura 7: Passos pré-laboratoriais. A: Indexação dos implantes 21 e 22. B: Moldagem de transferência e dos preparos das PPFs. C: Prova dos coppings em resina acrílica. D: Moldagem para remontagem dos coppings.

Foi realizada a prova da moldeira aberta e escolha da cor (A2) da cerâmica, pela escala Vita (Wilcos, RJ, Brasil). O silicone de adição densa foi manipulado e o fio \#00 foi removido, simultaneamente, à injeção do silicone de adição leve, no interior do sulco gengival, com auxílio do dispensador e da ponta automisturadora. Ao longo da moldagem com duplo fio, realizou-se, concomitantemente, a moldagem com transferentes quadrados (radiografados anteriormente) e com silicone de adição pesada e leve Express XT Soft (3M ESPE, MN, EUA), pela técnica da dupla mistura.

Ao retornar do laboratório foi realizada a prova dos copings em resina acrílica (Figura $7-\mathrm{C}$ ) para a avaliação inicial da adaptação cervical e do espaço oclusal. Após a fresagem da zircônia os copings foram novamente provados e radiografados, em seguida foi feita sua remontagem com silicone de adição Express XT Soft (3M ESPE, MN, EUA) pela técnica da dupla mistura (Figura $7-\mathrm{D}$ ). Com a prova da aplicação de dissilicato de lítio e sua sinterização, verificou-se a escolha de cor a partir da prova molhada, o formato e posicionamento dos dentes, a adaptação das próteses sobre implante e sobre dente, com exame físico e radiografia periapical. Os pontos de contato, foram evidenciados com carbono (Accu Film II - Parkell, NY, EUA), ajustados com ponta diamantada tronco-cônica 720G (JOTA, Rüthi, Suíça) em peça reta e baixa rotação, e reenviados ao laboratório para a aplicação do Glaze (Figura 8).

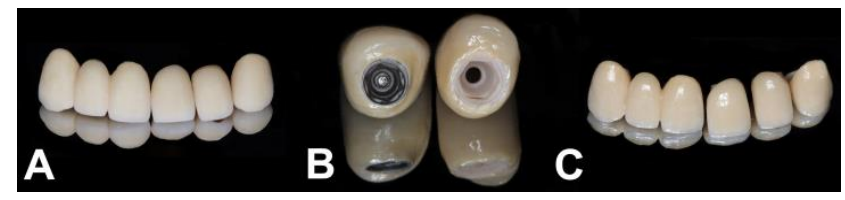

Figura 8: PPF, próteses sobre implante e prótese unitária. A: Vestibular das próteses sem Glaze. B: Visualização do coppings e da infraestrutura das próteses após sua finalização. C: Vestibular após a aplicação do Glaze.

Com a aprovação do paciente, após a prova das peças finalizadas, o cimento transparente foi selecionado previamente por meio de provas com o kit Try-In. Em seguida, iniciou-se o processo de cimentação como nas Figuras 9 e 10:

- Preparo do remanescente

1. Isolamento relativo;

2. Profilaxia com pedra pomes (SSWhite Duflex, RJ, Brasil) e escova de Robinson reta (American Burrs, $\mathrm{SC}$, Brasil) em todos os pilares;

3. Lavagem com jato de água e secos com jatos de ar e as próteses sobre implante do 21 e 22 posicionadas em boca;

4. Aplicação do ácido fosfórico (Condac 37, FGM Produtos Odontológicos Ltda, SC, Brasil) em dentina por 15 segundos;

5. Lavagem e secagem com algodão;

6. Aplicação do primer e adesivo Single Bond Universal (3M, Minnesota, EUA) com a ajuda de um Microbrush ${ }^{\circledR}$ Regular (2,0 mm);

7. Fotopolimerização por 20s (Bluephase N - Ivoclar Vivadent, SP, Brasil) em todas as faces.

- Preparo da peça protética

1. Jateamento com óxido de alumínio $(<50 \mu \mathrm{m})$

2. Lavagem e secagem com jatos de ar;

3. Silanização com Monobond N Universal (Ivoclar Vivadent, SP, Brasil) e Microbrush ${ }^{\circledR}$ Regular (2,0 $\mathrm{mm}$ );

4. Inserção do cimento resinoso dual Variolink ${ }^{\circledR} N$ (Ivoclar Vivadent, SP, Brasil) na cor Transparent com espátula $\mathrm{n}^{\circ} 1$ nas paredes axiais e levado em posição sob pressão positiva constante;

5. Remoção dos excessos após sua completa presa, realizada com 30s de fotopolimerização em cada pilar (Bluephase N - Ivoclar Vivadent, SP, Brasil);

As próteses implantossuportadas do 21 e do 22 foram levadas em posição, radiografadas e realizado sua estabilização final, do parafuso de retenção, com $15 \mathrm{~N}$ em torquímetro (TitaniumFix, São José dos Campos, SP, Brasil). Com a comprovação da adaptação, foi inserida fita para 
isolamento (TDV Dental, SC, Brasil), para proteger a cabeça do parafuso, e restaurado com resina composta de dentina Filtek ${ }^{\circledR}$ Z350 XT (3M ESPE, MN, EUA), na cor A2. Após a cimentação, foi avaliado o aspecto final da reabilitação a fim de mensurar a estética, fonética, função e conforto ${ }^{22}$ (Figura 11).

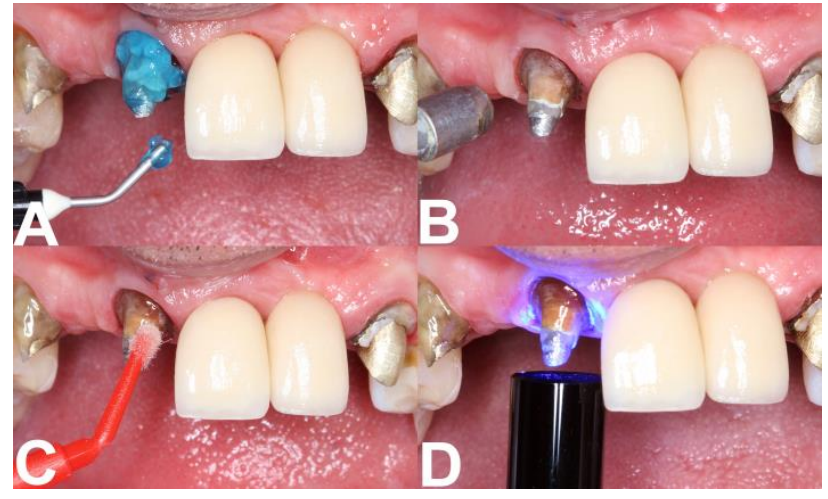

Figura 9: Processo prévio à cimentação da PPF ântero-superior A: Aplicação do ácido fosfórico $37 \%$ por 15 s. B: Lavando e secando os preparos. C: Aplicação do primer e adesivo. D: Fotopolimerização do primer e adesivo.

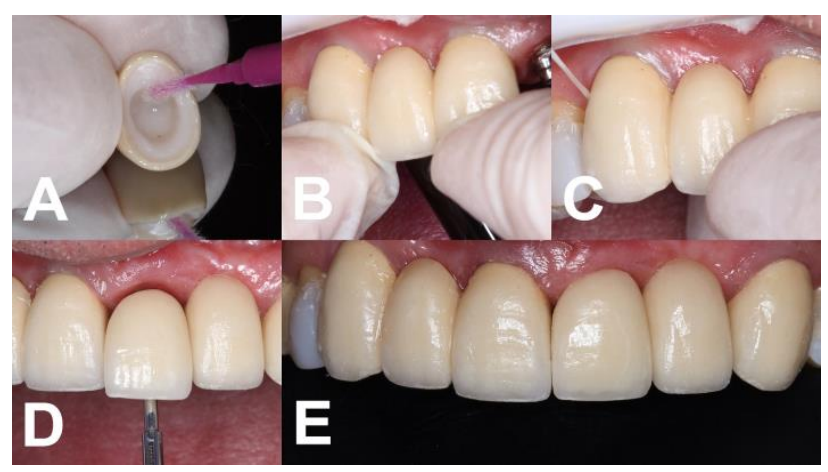

Figura 10 - Cimentação da PPF ântero-superior (A: Aplicação do silano. B: Levada a prótese em posição após a inserção do cimento. C: Remoção dos excessos de cimento das interproximais. D: Posicionamento da prótese parafusada implantossuportada para torque. E:Finalização da instalação das PPFs.)

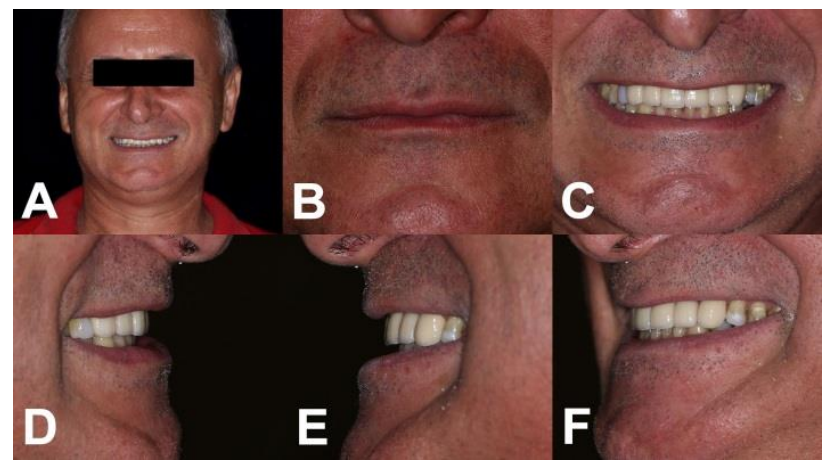

Figura 11: Fotografias extraorais de finalização do caso clínico. A: Frontal sorrindo. B: Frontal aproximado com lábios em repouso. C: Frontal aproximado sorrindo. D: Lateral $\left(90^{\circ}\right)$ direita sorrindo. E: Lateral $\left(90^{\circ}\right)$ esquerda sorrindo. F: Lateral $\left(45^{\circ}\right)$ esquerda sorrindo.

\section{DISCUSSÃO}

A avaliação do sorriso é uma importante etapa no diagnóstico, uma vez que esse é crucial para todo o tratamento odontológico estético ${ }^{3}$. No presente relato, o paciente apresentava desgastes na região anterior, com próteses apresentando desgastes incisais, dando aspecto de sorriso invertido, exibindo uma assimetria e a sensação de envelhecimento. Além disso, apresentava próteses desadaptadas na região cervical, mas sem exposição visual devido ao sorriso do paciente.

Para a confecção das PPFs na região anterior superior, foram envolvidas particularidades específicas estéticas e funcionais para a reabilitação oral do paciente. Dentre essas, visando um resultado harmonioso, foi necessária a reprodução das características intrínsecas e extrínsecas de cor, forma e textura superficial da dentição natural ${ }^{23}$.

Apesar do espaço oclusal presente na área do 21 ser reduzido e o implante não seguir o posicionamento ideal ${ }^{24}$, existem componentes protéticos e materiais que possuem propriedades físicas bastante vantajosos, como a liga de cobaltocromo ( $\mathrm{CoCr}$ ). A troca do componente UCLA pelo Esteticone fez-se necessário para que a vestibularização do 21 pudesse ser corrigida com a prótese sobre implante, uma vez que se fosse mantido o componente, a correção não seria possível. Já a combinação, da infraestrutura de $\mathrm{CoCr}$ e a cerâmica de cobertura de dissilicato de lítio, foi realizada devido à resistência e durabilidade empregados à ela ${ }^{25,26}$. A liga utilizada apresenta essas características devido a capacidade dos componentes metálicos inibirem qualquer reação subsuperficial ${ }^{25,26}$. Entretanto, sua característica óptica acinzentada e com baixa translucidez difere e contrasta grandemente das próteses puras de cerâmica, classificando-o como um material menos estético. Devido a atual evolução dos sistemas cerâmicos, são permitidas inúmeras associações de materiais. Entretanto, imitar as características visuais das próteses livres de metal em próteses metalocerâmicas é desafiador. Nesses casos, a seleção do material cerâmico influencia diretamente no resultado final ${ }^{23}$.

A estética do sorriso não é baseada apenas no formato, cor, posicionamento e textura dos dentes, nela também está envolvida a saúde e qualidade dos tecidos periodontais assim como a presença ou ausência de papilas e dos contornos gengivais ${ }^{27}$. Visando um resultado harmonioso tanto da estética branca quanto da rosa, foi realizado o enxerto conjuntivo aonde posteriormente foi instalado o pôntico do 12 em uma PPF. Contralateralmente não foi necessária a cirurgia periodontal, pois haviam implantes situados na área do incisivo central e lateral superiores, o que resultou em um defeito ósseo reduzido, quando levado em comparação ${ }^{28}$.

A combinação de próteses sobre implante e dentossuportadas em áreas estéticas requer atenção pois embora não seja provado estatisticamente que ao redor das próteses sobre implante ocorre uma redução da quantidade e qualidade da papila, essas frequentemente necessitam de enxertos gengivais para que ocorra uma melhora estética ${ }^{29,30}$. A perda do 
volume gengival é um grande desafio, especialmente quando se encontra em áreas anteriores. Seu condicionamento pode ajudar na formação de papilas reduzindo cirurgias periodontais ou então complementando-as, restabelecendo o contorno gengival adequado e melhorando a estética e a fonética do paciente ${ }^{31}$.

\section{CONCLUSÃO}

Apesar de serem inseridos em região estética, da disparidade dos materiais empregados e dos pilares, é possível mimetizá-los e oferecer resultados reabilitadores suficientemente satisfatórios, quando feito um correto diagnóstico e planejamento do caso clínico.

\section{REFERÊNCIAS}

1. Cardenas AFM, Mora CAP, Siqueira FSF, Parreiras SO, Gomes JC. Restabelecimento estético de um sorriso envelhecido: Caso clínico. Revista APCD de Estética. 2015; 3(1):42-52.

2. Strasding M, Fehmer V, Pjetursson BE, Sailer I. Extending the service life of existing dental restorations with esthetic and functional limitations. J Prosthet Dent. 2018;119(6):893-96.

3. Koidou VP, Rosenstiel SF, Rashid RG. Celebrity smile esthetics assessment: Smile angulation. J Prosthet Dent, 2017;117(5):636-41.

4. Levin, EI. Dental esthetics and the golden proportion. J Prosthet Dent. 1978;40(3):244-52.

5. Flores-Mir C, Silva E, Barriga MI, Lagravere MO, Major PW. Lay person's perception of smile aesthetics in dental and facial views. J Orthod. 2004;31(3):204-9.

6. Cotrim, ER, Vasconcelos Júnior, ÁV, Haddad, ACSS, Reis SAB. Perception of adults' smile esthetics among orthodontists, clinicians and laypeople. Dental Press J. Orthod. 2015; 20(1):40-4.

7. Chaudhari A, Bagga DK, Agrawal P, Kalra H, Sirohi D. An assessment of the self-satisfying smile among different professionals. J Int Oral Health. 2018;10(3):111-14.

8. Papaspyridakos P, Chen CJ, Singh M, Weber HP, Gallucci GO. Success criteria in implant dentistry: a systematic review. J Dent Res. 2012;91(3):24248.

9. Bonfante EA, Suzuki M, Lorenzoni FC, Sena LA, Hirata R, Bonfante $\mathrm{G}$ et al. Probability of survival of implant-supported metal ceramic and CAD/CAM resin nanoceramic crowns. Dent Mater J. 2015;31(8):e168-77.

10.Egilmez F, Ergun G, Cekic-Nagas I, Bozkaya S. Implant-supported hybrid prosthesis: conventional treatment method for borderline cases. Eur J Dent. 2015;9(3):442-48.
11.Schweitzer DM, Goldstein GR, Ricci JL, Silva NR, Hittelman EL. Comparison of bond strength of a pressed ceramic fused to metal versus feldspathic porcelain fused to metal. J Prosthodont. 2005;14(4):239-47.

12.Venkatachalam B, Goldstein GR, Pines MS, Hittelman EL. Ceramic pressed to metal versus feldspathic porcelain fused to metal: a comparative study of bond strength. Int $\mathrm{J}$ Prosthodont. 2009;22(1):94-100.

13.Holden JE, Goldstein GR, Hittelman EL, Clark EA. Comparison of the marginal fit of pressable ceramic to metal ceramic restorations. J Prosthodont. 2009;18: 645-48.

14. Sinhori BS, de Andrada MAC, Lopes GC, Monteiro Junior S, Baratieri LN. Influence of Teeth Preparation Finishing on the Adaptation of Lithium Disilicate Crowns. Int J Biomater. 2017; ID 2078526.

15.Hoppen LRC, Garbin CA, Rigo L, Schuh C, FederizzI L. Comparação estética entre coroas confeccionadas com os sistemas Cubo e metalocerâmico. Rev Sul-Bras Odontol. 2010;7(2):146-53.

16. Mazur CE, Machado CT, Malheiros Pfau VJ, Augusto Pfau, E. Planejamento multidisciplinar na reconstrução do sorriso. JCDR. 2017; 14(2):62-70.

17.Zuhr O, Bäumer D, Hürzeler $M$ The addition of soft tissue replacement grafts in plastic periodontal and implant surgery: critical elements in design and execution. J Clin Periodontol. 2014; 41(Suppl15):S123-42.

18.Consolaro A. Saucerização: um mecanismo natural de adaptação peri-implantar cervical. Dental Press Implantol, 2014;8(4):8-15.

19. Nealon FH. Acrylic restorations by the operative nonpressure procedure. J Prosthet Dent. 1952;2(4):513-27.

20.Newman MG, Takei H, Klokkevold PR, Carranza FA. Periodontia clínica. Rio de Janeiro: Elsevier Brasil; 2007. p.926-35.

21.Raetzke PB. Covering localized areas of root exposure employing the "envelope" technique. J Periodontol. 1985;56(7):397-402.

22. Hannah R, Ramani P, Sherlin HJ, Ranjith G, Ramasubramanian A, Jayaraj G et al. Awareness about the use, ethics and scope of dental photography among undergraduate dental students dentist behind the lens. RJPT. 2018; 11(3):1012-16.

23. Alberton SB, Alberton V, Carvalho RV. Providing a harmonious smile with laminate veneers for a patient with peg-shaped lateral incisors. J Conserv Dent. 2017;20(3):210-13.

24. Vervaeke S, Matthys C, Nassar R, Christiaens V, Cosyn J, De Bruyn H. Adapting the vertical position of implants with a conical connection in 
relation to soft tissue thickness prevents early implant surface exposure: A 2-year prospective intra-subject comparison. J Clin Periodontol. 2018;45(5):605-12.

25. Anusavice K, Shen C, Rawls HR. Dental casting and soldering alloys. In: Anusavice KJ, Phillips' Science of Dental Material. St. Louis: Elsevier; 2003. p.563-620.

26. Arinc H. Implant-supported fixed partial prostheses with different prosthetic materials: a three-dimensional finite element stress analysis. Implant Dent. 2018;27(3):303-10.

27. Monnet-Corti V, Antezack A, Pignoly M. Comment parfaire l'esthétique du sourire: toujours en rose! Orthod Fr. 2018;89(1):71-80.

28. Tonetti MS, Cortellini P, Graziani F, Cairo F, Lang NP, Abundo $\mathrm{R}$ et al. Immediate versus delayed implant placement after anterior single tooth extraction: the timing randomized controlled clinical trial. J Clin Periodontol. 2017; 44(2):215-24.

29. Pradeep AR, Karthikeyan BV. Peri-implant papilla reconstruction: Realities and limitations. J Periodontol. 2006; 77(3):534-44.

30. Nariman RH, Pai UY, Soumya MK, Hegde R. A clinical assessment of the volume of interproximal papilla after definitive prosthesis around immediate and delayed loading implants placed in the maxillary esthetic zone: An in vivo study. J Indian Prosthodont Soc. 2018;18(2):168-73.

31. Neves FDD, Silveira-Júnior CD, Coró V, SilvaNeto JP, Simamoto-Júnior PC, Prado CJD. Gingival conditioning in an implant-supported prosthesis: a clinical report. J Oral Implantol. 2013;39(4):483-85.

\section{CONFLITO DE INTERESSES}

Os autores declaram não haver conflitos de interesse.

AUTOR PARA CORRESPONDENCIA

\section{Aldieris Alves Pesqueira}

aldieris.pesqueira@unesp.br

Submetido em 25/09/2018

Aceito em 17/01/2019 